\title{
Short-Term Operation Scheduling in Renewable-Powered Microgrids: A Duality-Based Approach
}

\author{
Binyan Zhao, Student Member, IEEE, Yi Shi, Member, IEEE, Xiaodai Dong, Senior Member, IEEE, \\ Wenpeng Luan, Senior Member, IEEE, and Jens Bornemann, Fellow, IEEE
}

\begin{abstract}
This paper considers the operation scheduling problem in renewable-powered microgrids, which is used to determine the least-cost unit commitment (UC) and the associated dispatch, while meeting load, environmental, and system operating requirements. The intermittency nature of the renewable energy sources, as well as microgrid's capacity to operate either in parallel with, or autonomously of, the traditional power grid, pose new challenges to this classic optimization task. A probability-based concept, probability of self-sufficiency (PSS), is introduced to indicate the probability that the microgrid is capable of meeting local demand in a self-sufficient manner. Furthermore, to the best of our knowledge, we make the first attempt in approaching the mixed-integer UC problem from a convex optimization perspective, which leads to an analytical closed-form characterization of the optimal commitment and dispatch solutions. The simulation results show that 1) the proposed method achieves an efficient performance that incurs no loss of optimality with lower complexity than existing algorithms; 2) an energy storage system (ESS) with suitable capacity contributes to the self-sufficiency target of a microgrid, and the stored energy varies less remarkably as the microgrid tends to operate more independently; 3 ) the proposed method provides guidelines in deciding the ESS size to achieve a desired PSS.
\end{abstract}

Index Terms-Distributed generators, duality, economic dispatch, microgrid, renewable energy resources, unit commitment (UC).

\section{NOMENCLATURE}

Symbol
$\alpha_{(.)}, \beta_{(.)}, \gamma_{(.)}$
$\Delta_{d}$
$\Delta_{w}$
$\epsilon$
$\lambda_{(.)}, \mu_{(.)}, \nu_{(.)}$
$\mu_{e, d}$
$\mu_{e, w}$
$\rho_{(.)}$
$\sigma_{e, d}^{2}$
$\sigma_{e, w}^{2}$
$\tau^{(.)}$

\section{Description}

Emission coefficients of a unit.

Demand forecast error.

Wind power forecast error.

Convergence criterion parameter.

Lagrange multipliers.

Mean of demand forecast error.

Mean of wind power forecast error.

Cooling time constant of a unit.

Variance of demand forecast error.

Variance of wind power forecast error.

Step size of the subgradient method.

Manuscript received November 23, 2012; revised April 12, 2013 and July 15, 2013; accepted July 16, 2013. Date of publication September 30, 2013; date of current version December 12, 2013.

B. Zhao, Y. Shi, X. Dong, and J. Borenmann are with the Department of Electrical and Computer Engineering, University of Victoria, Victoria, BC V8W 3P6, Canada (e-mail: binyanz@ece.uvic.ca; shiyipfc@ece.uvic.ca; xdong@ece. uvic.ca; jbornema@ece.uvic.ca).

W. Luan is with the State Grid Smart Grid Research Institute, Beijing 100085, China (e-mail: luanwenpeng@sgri.sgcc.com.cn).

Color versions of one or more of the figures in this paper are available online at $\mathrm{http}: / /$ ieeexplore.ieee.org.

Digital Object Identifier 10.1109/TSTE.2013.2279837

\begin{tabular}{|c|c|}
\hline$\varphi_{(.)}$ & UC indication function. \\
\hline & Emission limit. \\
\hline$[x]_{b}^{a}$ & $\begin{array}{l}\text { Euclidean projection of } x \text { to the interval } \\
{[a, b] \text {, i.e., }[x]_{b}^{a}=\max (\min (x, a), b) \text {. }}\end{array}$ \\
\hline$(x)^{+}$ & $\begin{array}{l}\text { Euclidean projection of } x \text { to the interval } \\
{[0,+\infty) \text {, i.e., }(x)^{+}=\max (x, 0)}\end{array}$ \\
\hline$a_{(.)}, b_{(.)}, c_{(.)}$ & Fuel cost coefficients of a unit. \\
\hline$b_{(.)}^{\prime}, c_{(.)}^{\prime}$ & Aggregated cost coefficients of a unit. \\
\hline & Maintenance cost coefficients of a unit. \\
\hline $\operatorname{erf}()$. & Error function [17, eq. 8.250.1]. \\
\hline$i$ & Unit index (subscript). \\
\hline$n$ & Iteration index (superscript). \\
\hline$p_{(.)}$ & Power dispatch of a unit. \\
\hline$p_{(.)}^{*}$ & Optimal power dispatch of a unit. \\
\hline$p_{(.)}^{\min }, p_{(.)}^{\max }$ & $\begin{array}{l}\text { Minimum and maximum power generation } \\
\text { limits. }\end{array}$ \\
\hline$p_{w}$ & Wind turbine power output. \\
\hline $\bar{p}_{(.)}$ & $\begin{array}{l}\text { Power dispatch of a unit in the relaxed UC } \\
\text { problem. }\end{array}$ \\
\hline$\hat{p}_{w, t}$ & Forecasted wind power in time interval $t$. \\
\hline$t$ & Hour index (subscript). \\
\hline$u_{(.)}$ & $\begin{array}{l}\text { Unit status indicator of a unit where } 1 \text { means } \\
\text { ON and } 0 \text { means OFF. }\end{array}$ \\
\hline$v_{\text {in }}$ & Cut-in wind speed. \\
\hline$v_{\text {out }}$ & Cut-out wind speed. \\
\hline$v_{r}$ & Rated wind speed. \\
\hline$v_{w}$ & Wind speed. \\
\hline$w_{r}$ & Rated electrical power. \\
\hline$A_{t}$ & Modified electricity demand in time interval $t$. \\
\hline$C_{\min }, C_{\max }$ & $\begin{array}{l}\text { Lower and upper limits of the energy stored } \\
\text { in ESS. }\end{array}$ \\
\hline $\mathrm{CSC}_{(.)}$ & Cold start-up cost of a unit. \\
\hline$D()$. & Dual function. \\
\hline$D_{t}$ & Forecasted demand in time interval $t$. \\
\hline$E_{(.)}$ & Emission function of a unit. \\
\hline$F_{\Delta_{w}}$ & $\mathrm{CDF}$ of wind power forecast error. \\
\hline $\mathrm{FC}_{(.)}()$. & Fuel cost function of a unit. \\
\hline $\mathrm{HSC}_{(.)}$ & Hot start-up cost of a unit. \\
\hline $\mathcal{L}()$. & Lagrange function. \\
\hline $\mathrm{MC}_{(.)}()$. & Maintenance cost function of a unit. \\
\hline $\operatorname{MUT}_{(.)}, \operatorname{MDT}_{(.)}$ & $\begin{array}{l}\text { The time a unit needs to remain ON/OFF if ON/ } \\
\text { OFF at the beginning of the scheduling period. }\end{array}$ \\
\hline$N$ & Total number of units. \\
\hline$P()$. & Probability operator. \\
\hline PSS & Probability of self-sufficiency. \\
\hline
\end{tabular}


$R_{t}$

$\operatorname{STC}_{(.)}$

$T$

TC

$\mathrm{TU}_{(.)}, \mathrm{TD}_{(.)}$
Operating reserve requirement in time interval $t$.

Start-up cost function of a unit.

Total time horizon of 1 day.

Total cost of all units.

The time a unit has been on/OFF at the beginning of the scheduling period.

\section{INTRODUCTION}

S USTAINABILITY has become an imperative requirement on the electric power infrastructure with the impending energy crisis and environment deterioration. In parallel to the rapid evolution of smart-grid-based solutions [1], another emerging power distribution system known as microgrids is also quietly gaining momentum. A microgrid is an integrated system consisting of a set of distributed generators (DGs) [microturbines (MT), fuel cells (FC), reciprocating engines] and renewable energy sources (RESs) [solar photovoltaics (PV) and wind turbines (WT) systems] [2], which function cooperatively to furnish the cooperation in parallel with, or autonomously of, the traditional electricity macrogrid. The defining characteristic of a microgrid is its ability to separate itself seamlessly from the macrogrid during a utility grid disturbance, and function as a self-controlled entity with high efficiency and low greenhouse gas emissions. This transparent adaptation in operational modes, along with the capacity to better manage distributed energy resources (DERs), renders microgrid the most promising solution to develop a more reliable and decentralized energy system.

The daily operation of a microgrid involves finding the leastcost dispatch of the DGs that minimizes the total operating cost, while meeting the electrical load and satisfying various technical, environmental, and operating constraints. This can be seen as a downsized version of the unit commitment (UC) problem that is traditionally applied to large central generators in macrogrid [3]. Mathematically, the UC task can be described as a mixed-integer optimization problem with a nonlinear solution space, which has been the subject of intensive investigation for more than 40 years (c.f., bibliographical survey [4]). Nevertheless, the unique features of microgrids introduce further restrictions as well as simplifications to this classic optimization task that needs to be addressed.

The biggest challenge comes from the intermittent nature of RESs, which often leads to power variations and makes it much more difficult to produce accurate day-ahead schedules in microgrids. Therefore, the operation scheduling of the dispatchable DGs should be performed at a much finer level, providing quick and continuous power provision, based on the most recent and accurate forecasted data [5]. Although large thermal units are often subject to ramping rate limits that are typically in the order of several tens of megawatts per hour, it only takes several minutes for an MT to ramp up from 0 to full load [6]. This further supports the operation scheduling to be performed at a more granular level for microgrids [7]. Last but not the least, the capacity of switching between different operational modes calls for a proper modeling framework that reflects the unique characteristic of microgrids.

Various numerical-based algorithms, including some typical heuristic methods such as genetic algorithm (GA) [8], [9], particle swarm optimization (PSO) [10], simulated annealing techniques [11], [12], and network-flow programming [13], have been proposed to solve the optimal UC problem, but most of them only provide a reasonable numerical solution (suboptimal, nearly global optimal) and have high computational complexity. For the classical mixed-integer UC problem, the branch and bound (BB) technique provides exact accurate numerical results. However, the procedure is generally not efficient except when large portions of the solution space can be quickly discarded in the case that there are not too many solutions having near optimal function values. In [14], a linear optimization problem was formulated and numerically solved to determine the optimal size of energy storage system (ESS) that minimizes the operating cost. The authors in [15] and [7] also established UC strategies based on piecewise linear blocks approximating the quadratic objective function and frequency droop scheme. The authors in [16] modeled explicitly the length of time the microgrid operates autonomously and used a Monte Carlo analysis to study the impact of RESs over a set of United Kingdom commercial load profiles.

This paper deals with a quadratic formulation of the environmental/economic UC optimization problem in a microgrid that consists of DGs, RESs, and an ESS. The contributions of this work are summarized as follows: 1) in light of microgrid's unique operational feature and to take into account the forecast errors that exist in demand and wind power forecast, a novel probability-based concept is proposed to indicate the probability that the microgrid is able to operate in islanded mode, termed "probability of self-sufficiency" (PSS); 2) the mixed-integer UC problem is approached from a convex optimization perspective, which leads to an analytical closed-form solution. Compared to two classical methods, BB and GA, the proposed method uses significantly less processing time yet renders no loss of optimality in performance; and 3) the proposed method provides guidelines in deciding the size of ESS to improve the autonomous target PSS for a microgrid efficiently.

The remainder of this paper is organized as follows. Section II discusses the basic system models including wind power forecast, operating cost, emission, as well as various unit and operation constraints. In Section III, the optimal UC problem is formulated, transformed into a convex optimization problem, and finally solved in analytical closed forms. An efficient subgradient-based algorithm is also proposed to obtain the optimal UC solution in this section. Section IV conducts numerical simulations that verify the efficiency and reliability of the proposed scheme. Finally, concluding remarks are drawn in Section V.

\section{SyStem Model}

We consider a microgrid that consists of a set of $N$ DG units, including both MTs and FCs, an RES, the WT, and an energy storage module, the ESS. Electrical loads in the microgrid are prioritized into tiers, which consists of critical loads that relate to essential processes that must be always met and lower-priority non-critical loads that can be temporarily removed until adequate power is available. Due to the limited capacity of the DERs and as in [18]-[20], we assume that the microgrid schedules its units to meet the critical loads in the highest priority, satisfies the 
non-critical loads using best efforts, and purchases power from the macrogrid in case of supply shortage. In addition, to better utilize an environmentally friendly resource, we assume that the WT is always on and functions as the primary power source [14]. The DGs, on the other hand, serve as backup generators and work collaboratively with the WT to meet the critical loads. The ESS module is introduced to mitigate the renewable power intermittencies and load mismatches. In this work, we assume that the microgrid updates its UC strategy every $1 \mathrm{~h}^{1}$ during which load and generation are considered constant.

\section{A. Forecasted Wind Power Model}

Wind power is the electrical power generated by WT, installed in locations with strong and sustained winds. In practice, the actual wind power $p_{w}$ almost entirely depends on the wind speed $v_{w}$ when other physical limitations are fixed or changed relatively slowly [14]. In principle, $v_{w}$ is a random variable and varies continuously over time. In this work, we assume that $v_{w}$ remains unchanged in one scheduling period (i.e., $1 \mathrm{~h}$ ), and can vary independently between different scheduling periods.

Extensive research has been done in developing wind forecasting models and approaches [14], [21]. To capture the relationship between the wind speed and wind power and as in [14] and [22], the following piecewise linear model is adopted:

$$
p_{w}= \begin{cases}w_{r}, & v_{r} \leq v \leq v_{\text {out }} \\ \frac{\left(v-v_{\text {in }}\right) w_{r}}{v_{r}-v_{\text {in }}}, & v_{\text {in }} \leq v<v_{r} \\ 0, & \text { else. }\end{cases}
$$

\section{B. Cost Models for MT and FC}

MTs are small electricity generators that burn gaseous and liquid fuels to create high-speed rotation that turns an electrical generator. Depending on the size range, an MT can ramp up from 0 to full load between $10 \mathrm{~s}$ to several minutes [6]. The FC technology uses an electrochemical process rather than a combustion process to generate electricity. Polymer electrolyte FCs, also known as proton exchange membrane FCs, are particularly attractive for microgrids that require rapid start-up and quick response to load change [23].

The operating cost of an MT/FC usually includes fuel cost, maintenance cost, and start-up cost.

Fuel Costs: The fuel costs for DGs are considered as a quadratic model [24], which includes linear fuel cost models as special cases [7], [14]. The fuel costs of unit $i$ in time interval $t$ can be expressed as

$$
\mathrm{FC}_{i, t}\left(p_{i, t}\right)=a_{i} p_{i, t}^{2}+b_{i} p_{i, t}+c_{i}
$$

Maintenance Costs: The maintenance costs for DGs are based on forecasts with minimal real-life situations, which are assumed to be proportional to the produced power [25]. Therefore, the maintenance costs of unit $i$ in time interval $t$ is

\footnotetext{
${ }^{1}$ Depending on different application requirements, a smaller updating interval can be selected since the closed-form UC solutions can be computed fast.
}

$$
\mathrm{MC}_{i, t}\left(p_{i, t}\right)=d_{i} p_{i, t} .
$$

Start-Up Costs: The generator start-up cost depends on the time the unit has been off prior to a start-up. The start-up cost of unit $i$ in time interval $t$ can be represented by an exponential cost curve [24, eq. (3.12)]

$$
\mathrm{STC}_{i, t}=\left(\mathrm{HSC}_{i}+\mathrm{CSC}_{i}\left[1-\exp \left(\frac{-\mathrm{TD}_{i, t}}{\rho_{i}}\right)\right]\right) \cdot\left(1-u_{i, t-1}\right)
$$

Sometimes, the industry is interested in the total cost per day

$$
\mathrm{TC}=\sum_{t=1}^{T} \sum_{i=1}^{N} u_{i, t}\left(\mathrm{FC}_{i, t}+\mathrm{MC}_{i, t}+\mathrm{STC}_{i, t}\right) .
$$

\section{Emission Model}

Emission effects should be taken into account for an environmentally friendly power production. The microgrids are envisioned to be new energy savings and green grids in the future, which entails carbon emissions limited to regulations and legal requirements.

The amount of emissions produced depends on the fuel used, pollution control devices installed, and the amount of electricity generated. In this work, we assume that only DGs produce emissions and the RESs are emission free. ${ }^{2}$ The emission function is typically expressed as a polynomial, the order of which depends on the desired accuracy [27], [28]. As in [15] and [28], a quadratic function is considered for the emission curve as follows:

$$
E_{i, t}\left(p_{i, t}\right)=\alpha_{i} p_{i, t}^{2}+\beta_{i} p_{i, t}+\gamma_{i}
$$

\section{Energy Storage System}

In renewable-powered microgrids, the problem of mitigating power intermittencies and load mismatches is an important and challenging task. In this context, an ESS plays a critical role in shaving peak demand and compensating forecast errors. For instance, when the forecasted wind power is smaller than the actual value (i.e., an underestimate), the supplied power is likely to be larger than the actual electricity demand, in which case the ESS will be functioning in charging state to store surplus electrical/renewable energy, which can be dispatched properly later in the event of power shortage.

The charge and discharge of the ESS is subject to stored energy limits, $C_{\min }$ and $C_{\max }$, which specify the minimum and maximum energy stored in the battery bank, respectively. In this case, $C_{\max }$ is set as the full capacity of the ESS and $C_{\min }$ to be around $10 \%$ of its full capacity. The ESS is also subject to starting and ending limits that specify the initial and final energy inside the battery bank during the course of 1 day. In this work, the starting and ending limits are both selected as $C_{\min }$ for the purpose of energy balance of the ESS [14].

\footnotetext{
${ }^{2}$ Note that the emissions in the production process of WT and other equipments [26] have not been considered.
} 


\section{E. Unit and Operation Constraints}

Maximum and Minimum Output Limits: The output power of the DG in stable operation is restricted by its lower and upper limits as follows:

$$
p_{i}^{\min } \leq p_{i, t} \leq p_{i}^{\max }
$$

In our case, the minimum available power for the DGs is 0 , i.e., the DG can be turned ofF when the output power from the WT is enough to meet the demanded power.

Minimum Up/Down Time: Once a DG is switched on, it has to operate continuously for a certain number of times before it can be switched off. Also, a certain number of hours have to pass before a DG can be brought online after being switched ofF. Violation of such constraints can shorten the unit's life time. Mathematically, we have

$$
\begin{aligned}
& \left(\mathrm{TU}_{i, t-1}-\mathrm{MUT}_{i}\right)\left(u_{i, t-1}-u_{i, t}\right) \geq 0 \\
& \left(\mathrm{TD}_{i, t-1}-\mathrm{MDT}_{i}\right)\left(u_{i, t}-u_{i, t-1}\right) \geq 0 .
\end{aligned}
$$

For DGs in microgrids, the minimum up/down time of DGs is around 600 and $300 \mathrm{~s}$ [5], respectively, which is always satisfied under hourly scheduling operations.

Ramp Rates: Traditional thermal units are often subject to ramp rate limits that specify the amount a unit's generation can increase or decrease during one scheduling period. In the context of microgrids, small DG units can ramp up from 0 to full load in the order of several minutes [23]. Thus, the ramp rate limits are typically not reached under normal hourly scheduling operations.

Emission Limits: To comply with the purpose of environment conservation and reduce the greenhouse gas footprint, we impose hourly emission limits on all the DGs. Mathematically, we have

$$
\sum_{i=1}^{N} u_{i, t} E_{i, t}\left(p_{i, t}\right) \leq \zeta
$$

Operating Reserves: In the event of a power supply disruption, operating reserve constraints guarantee that there exist extra generating capacity to the system that can be brought online immediately (spinning reserves) or within a short interval (supplementary reserves). In microgrids with fast-start DGs, operating reserves are imposed as follows [3]:

$$
\sum_{i=1}^{N}\left(p_{i}^{\max }-u_{i, t} p_{i, t}\right) \geq R_{t} .
$$

Probability of Self-Sufficiency: Both demand and renewable power forecast are prone to errors, which negatively affect the microgrid in meeting the local power demand and their autonomous and independent functions. Once a microgrid cannot meet the power demand solely based on local generating units, it can switch to a grid-connected mode and purchase energy from the upstream macrogrid. To better understand the impacts of operational mode on total operating cost, we propose the use of a novel probability-based concept, PSS, which indicates the target probability that the microgrid is able to operate in islanded mode without purchasing energy from the macrogrid.
As in [29] and [30], we assume that both demand forecast error $\Delta_{d}$ and wind power forecast error $\Delta_{w}$ can be modeled as independent normally distributed random variables, i.e., $\Delta_{d} \sim N\left(\mu_{e, d}, \sigma_{e, d}^{2}\right)$ and $\Delta_{w} \sim N\left(\mu_{e, w}, \sigma_{e, w}^{2}\right)$, respectively. Then, the probabilistic power balance constraint can be expressed as

$$
P\left(\sum_{i=1}^{N} p_{i, t} u_{i, t}+\hat{p}_{w, t}+\Delta_{w} \geq D_{t}+\Delta_{d}\right) \geq \operatorname{PSS}
$$

which, after some algebra, can be reformulated as

$$
\begin{aligned}
\sum_{i=1}^{N} p_{i, t} u_{i, t} \geq A_{t}= & D_{t}-\hat{p}_{w, t}-\left(\left(\mu_{e, w}-\mu_{e, d}\right)\right. \\
& \left.+\sqrt{2\left(\sigma_{e, w}^{2}+\sigma_{e, d}^{2}\right)} \operatorname{erf}^{-1}(1-2 \mathrm{PSS})\right)
\end{aligned}
$$

\section{PRoblem Formulation AND Closed-Form Solutions}

Based on the aforementioned cost models and system constraints, we first formulate the UC optimization problem that is used to determine the loads and commitments of DGs such that the total cost is minimized, power demand is met, and the system constraints are satisfied. A duality-based analysis is then applied to derive closed-form solutions. Finally, an efficient subgradientbased method is introduced to numerically compute the optimal solutions.

\section{A. Problem Formulation}

The UC optimization problem in a particular operation scheduling interval $t$ can be written as

$$
\begin{array}{r}
\text { P1 } \min _{\substack{p_{i, t}, u_{i, t} \\
\forall i=1, \ldots, N}} \sum_{i=1}^{N} u_{i, t}\left(\mathrm{FC}_{i, t}+\mathrm{MC}_{i, t}+\mathrm{STC}_{i, t}\right) \\
\text { s.t. } \quad(7)-(11) \text { and }(13) \quad \forall i=1, \ldots, N .
\end{array}
$$

Evidently, problem $\mathbf{P 1}$ is a mixed-integer programming problem with a nonlinear solution space. In order to transform P1 into a convex optimization problem, we introduce auxiliary power variables $q_{i, t}=p_{i, t} u_{i, t}$ and relax $u_{i, t}$ to be a continuous variable in $[0,1]$. Thus, the transformed problem can be written as ${ }^{3}$

$$
\begin{aligned}
& \text { P2 } \min _{\substack{q_{i, t}, u_{i, t} \\
\forall i=1, \ldots, N}} \sum_{i=1}^{N}\left(a_{i} q_{i, t}^{2} / u_{i, t}+b_{i}^{\prime} q_{i, t}+c_{i, t}^{\prime} u_{i, t}\right) \\
& \text { s.t. } \sum_{i=1}^{N} q_{i, t} \geq A_{t} \\
& \quad \sum_{i=1}^{N}\left(\alpha_{i} q_{i, t}^{2} / u_{i, t}+\beta_{i} q_{i, t}+\gamma_{i} u_{i, t}\right) \leq \zeta \\
& \sum_{i=1}^{N}\left(p_{i}^{\max }-q_{i, t}\right) \geq R_{t} \\
& u_{i, t} p_{i}^{\min } \leq q_{i, t} \leq u_{i, t} p_{i}^{\max } \quad \forall i=1, \ldots, N
\end{aligned}
$$

\footnotetext{
${ }^{3}$ The objective function is defined at $u=0$ by continuity as $\lim _{u \rightarrow 0}\left(a q^{2} / u+b q+c u\right)=0$.
} 
where the aggregated cost coefficients are $b_{i}^{\prime}=b_{i}+d_{i}$ and $c_{i, t}^{\prime}=c_{i}+\mathrm{STC}_{i, t}$. Note that the objective function of $\mathbf{P 2}$ is equivalent to that of $\mathbf{P} 1$ as the change of variables is invertible except for $u_{i, t}=0$. The same statement holds for the emission constraints (10) and (14). In case that $u_{i, t}=0$, every $p_{i, t}$ solves P1.

The Hessian matrices of the objective function and the constraints of $\mathbf{P 2}$ are positive semi-definite, and the form of the whole problem meets the requirement of a convex problem [31], indicating that $\mathbf{P 2}$ is a convex problem.

\section{B. Closed-Form Economic Dispatch and UC Solutions}

To solve $\mathbf{P 2}$ analytically, we first decouple the optimization variables $q_{i, t}$ and $u_{i, t}$ by substituting $\bar{p}_{i, t}:=q_{i, t} / u_{i, t}$. Note that $\bar{p}_{i, t}=p_{i, t}$ for the case of interest, i.e., $u_{i, t} \neq 0$. For this reason, we will henceforth use $\bar{p}_{i, t}=p_{i, t}$. The Lagrangian function can then be written as

$$
\begin{aligned}
\mathcal{L}\left(\lambda_{t}, \mu_{t}, \nu_{t}, \mathbf{p}, \mathbf{u}\right)= & \sum_{i=1}^{N} u_{i, t} \varphi_{i, t}\left(p_{i, t}\right)+\lambda_{t} A_{t}-\mu_{t} \zeta \\
& +\nu_{t} R_{t}-\nu_{t} \sum_{i=1}^{N} p_{i}^{\max }
\end{aligned}
$$

where $\varphi_{i, t}\left(p_{i, t}\right)$ can be viewed as the UC indication function

$$
\begin{aligned}
\varphi_{i, t}\left(p_{i, t}\right)= & \left(a_{i}+\mu_{t} \alpha_{i}\right) p_{i, t}^{2}+\left(b_{i}^{\prime}+\mu_{t} \beta_{i}-\lambda_{t}+\nu_{t}\right) p_{i, t} \\
& +\left(c_{i, t}^{\prime}+\mu_{t} \gamma_{i}\right) .
\end{aligned}
$$

The dual function can be obtained by minimizing the Lagrangian (14), which is given by

$$
\begin{aligned}
D\left(\lambda_{t}, \mu_{t}, \nu_{t}\right)= & \min _{0 \leq u_{i, t} \leq 1} \sum_{i=1}^{N} u_{i, t} \min _{p_{i}^{\min } \leq p_{i, t} \leq p_{i}^{\max }} \varphi_{i, t}\left(p_{i, t}\right) \\
& +\lambda_{t} A_{t}-\mu_{t} \zeta+\nu_{t} R_{t}-\nu_{t} \sum_{i=1}^{N} p_{i}^{\max }
\end{aligned}
$$

The initial step to solve (16) is to tackle the inner minimization, the solution of which can be easily derived as

$$
p_{i, t}^{*}=\underset{p_{i}^{\min } \leq p_{i, t} \leq p_{i}^{\max }}{\arg \min } \varphi_{i, t}\left(p_{i, t}\right)=\left[-\frac{b_{i}^{\prime}+\mu_{t} \beta_{i}-\lambda_{t}+\nu_{t}}{2\left(a_{i}+\mu_{t} \alpha_{i}\right)}\right]_{p_{i}^{\min }}^{p_{i}^{\max }} .
$$

The next step is to solve the outer minimization over $u_{i, t}$ in (16), given the optimal power dispatch solution (17). Evidently, the minimum value is attained by setting $u_{i, t}=1$ for all $\varphi_{i, t}\left(p_{i, t}^{*}\right)<0$ and $u_{i, t}=0$ otherwise. Mathematically, we have

$$
u_{i, t}^{*}= \begin{cases}1, & \varphi_{i, t}\left(p_{i, t}^{*}\right)<0 \\ 0, & \text { otherwise }\end{cases}
$$

Remark 1: An important step in the course of analytical derivation is to decouple the optimizations of the commitment status $u_{i, t}$ and the power dispatch $p_{i, t}$ separately as in (16).
Besides, we observe that the commitment decisions are completely determined by the sign of $\varphi_{i, t}($.$) at the optimal$ dispatch $p_{i, t}^{*}$ [c.f. (18)]. Thus, $\varphi_{i, t}($.$) can be seen as a UC$ indication function. In the case that the power generated by the WT is sufficient to meet local power demand, the microgrid will be entirely powered by the RES and no DG needs to be turned on, i.e., $u_{i, t}=0$ for all $i$.

\section{Subgradient-Based Algorithm}

So far, we have found the analytical optimal solutions $p_{i, t}^{*}$ in (17) and $u_{i, t}^{*}$ in (18), which are, however, functions of the Lagrangian multipliers. By convexity, it suffices to obtain the optimal dual variables $\left(\lambda_{t}^{*}, \mu_{t}^{*}, \nu_{t}^{*}\right)$ to the dual problem, which are used to compute optimal primal solutions (17) and (18). The complete algorithm is formally stated in Algorithm 1. This section discusses the use of a subgradient-based iterative procedure to numerically compute the corresponding optimal Lagrangian multipliers $\lambda_{t}^{*}, \mu_{t}^{*}$, and $\nu_{t}^{*}$. The key iteration steps are [31]

$$
\begin{aligned}
& \lambda_{t}^{(n+1)}=\left[\lambda_{t}^{(n)}+\tau^{(n)}\left(A_{t}-\sum_{i=1}^{N} u_{i, t}^{*(n)} p_{i, t}^{*(n)}\right)\right]^{+} \\
& \mu_{t}^{(n+1)}=\left[\mu_{t}^{(n)}+\tau^{(n)}\left(\sum_{i=1}^{N} u_{i, t}^{*(n)} E_{i, t}\left(p_{i, t}^{*(n)}\right)-\zeta\right)\right]^{+} \\
& \nu_{t}^{(n+1)}=\left[\nu_{t}^{(n)}+\tau^{(n)}\left(R_{t}-\sum_{i=1}^{N}\left(p_{i}^{\max }-p_{i, t}^{*(n)} u_{i, t}^{*(n)}\right)\right)\right]^{+}
\end{aligned}
$$

which are provably convergent to the optimal value provided that the step sizes are selected to satisfy $\sum_{n=1}^{\infty} \tau^{(n)}=\infty$ and $\sum_{n=1}^{\infty}\left(\tau^{(n)}\right)^{2}<\infty$ [32]. A graphical convergence illustration can be found in the simulation section.

\section{Algorithm 1 Subgradient-based algorithm}

Initialize Lagrangian multiplier $\lambda_{t}^{(0)}, \mu_{t}^{(0)}$, and $\nu_{t}^{(0)}$ to arbitrary non-negative values and set the step size $\tau^{(n)}=1 / \sqrt{n+1}$.

\section{repeat}

Compute economic dispatch $p_{i, t}^{*(n)}$ using (17).

Obtain UC indication function $\varphi_{i, t}^{(n)}\left(p_{i, t}^{*(n)}\right)$ via (15).

Find unit status indicator $u_{i, t}^{*(n)}$ using (18).

Update $\lambda_{t}^{(n+1)}, \mu_{t}^{(n+1)}$, and $\nu_{t}^{(n+1)}$ via (19), (20), and (21), respectively.

until $\left|T C^{(n)}-T C^{(n-1)}\right|<\epsilon T C^{(n-1)}$.

\section{SimUlation Results AND Discussion}

In this study, a microgrid system consisting of two MTs, FC and WT, and one ESS is considered for a scheduling time horizon of $24 \mathrm{~h}$. The fuel cost coefficients, the emission coefficients, and the power limits of the DGs are summarized in Table I, where $p_{(.)}^{\min }$ of all DGs are $0 \mathrm{~kW}$. Similar parameter settings have also 
TABLE I

Parameters of Distributed Generators

\begin{tabular}{|c|c|c|c|c|c|c|c|}
\hline Unit & $a_{(.)}$ & $b_{(.)}$ & $c_{(.)}$ & $\alpha_{(.)}$ & $\beta_{(.)}$ & $\gamma_{(.)}$ & $p_{(.)}^{\max }(\mathrm{kW})$ \\
\hline MT1 & 20 & 50 & 100 & 6.49 & -5.55 & 4.09 & 30 \\
MT2 & 100 & 40 & 140 & 5.64 & -6.05 & 2.54 & 75 \\
FC & 10 & 20 & 20 & 3.38 & -3.55 & 5.33 & 100 \\
& & & & $\times 10^{-4}$ & $\times 10^{-4}$ & $\times 10^{-4}$ & \\
\hline
\end{tabular}

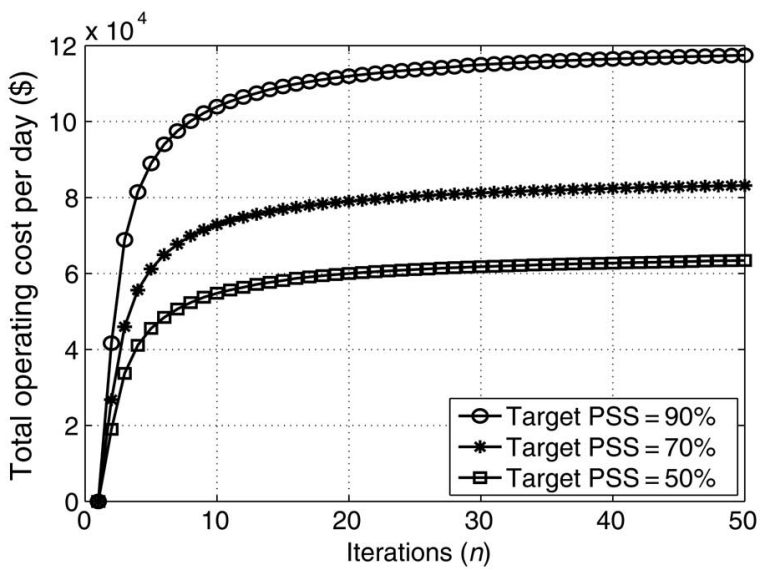

Fig. 1. Total operating cost per day under different PSS targets.

been used in [8]. The emission limit $\zeta$ is set as $150 \mathrm{~kg} / \mathrm{hr}$. The wind speed data samples adopted in this work are from the "Wind Test Center" in West Texas A\&M University [33], with the parameters in (1), $v_{\text {in }}=3.5 \mathrm{~m} / \mathrm{s}, v_{r}=14 \mathrm{~m} / \mathrm{s}$, and $v_{\text {out }}=25 \mathrm{~m} / \mathrm{s}$. Unless stated otherwise, the demand forecast error statistics and those of the wind power are set as $\mu_{e, d}=\mu_{e, w}=0, \sigma_{e, d}^{2}=144$, and $\sigma_{e, w}^{2}=256$. To understand the impact of PSS on the total operating cost, three different operation scenarios are considered, which are PSS $=90 \%$, $\mathrm{PSS}=70 \%$, and $\mathrm{PSS}=50 \%$.

\section{A. UC, Dispatching and Methods Comparison}

This section illustrates the derived UC solutions without considering the functionality of the ESS. The effects of ESS will be investigated in Section IV-C. Fig. 1 illustrates the fast convergence behavior of the proposed algorithm in optimizing the total operating cost per day of the microgrid. It takes only about 20 iterations to reach the optimal solution under all three PSS values. Besides, it is observed from Fig. 1 that the operating cost increases as the microgrid functions more autonomously, since more power has to be generated to ensure self-sufficiency and to mitigate demand and wind power forecast errors.

Fig. 2 shows the comparison results of four different methods in our environmental/economic dispatch optimization problem. We use GA and the proposed method (Prop-algor) to solve the original problem $\mathbf{P} \mathbf{1}$ and the transformed problem $\mathbf{P} 2$, respectively. In addition, $\mathrm{BB}$ is applied to solve $\mathbf{P} \mathbf{1}$, providing us an accurate result as a benchmark. In terms of computational complexity, the proposed method has $\mathbf{O}(N \log (1 / \epsilon))$ [31] time complexity with $N$ the number of generators in the system, while BB with depth-first search requires the computation on the

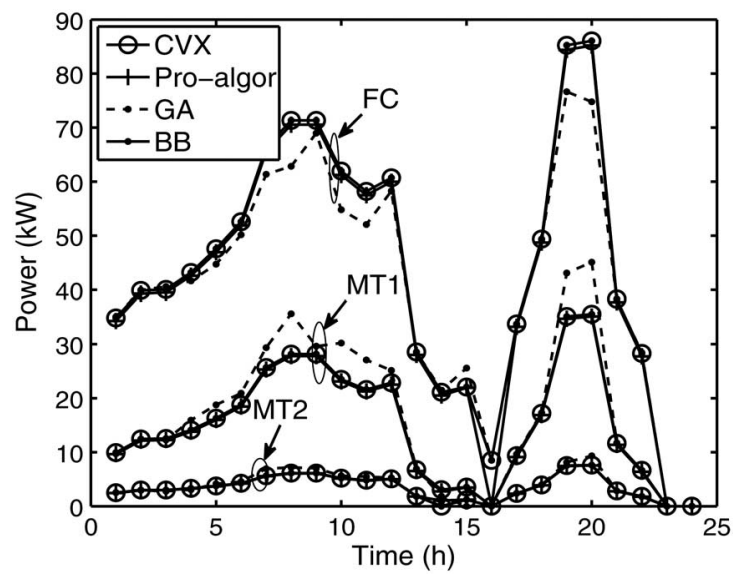

Fig. 2. Performance comparison of different optimization algorithms under PSS $=90 \%$. Population size $=200$, accuracy $=10^{-3}$, and generation $=2$ for GA.

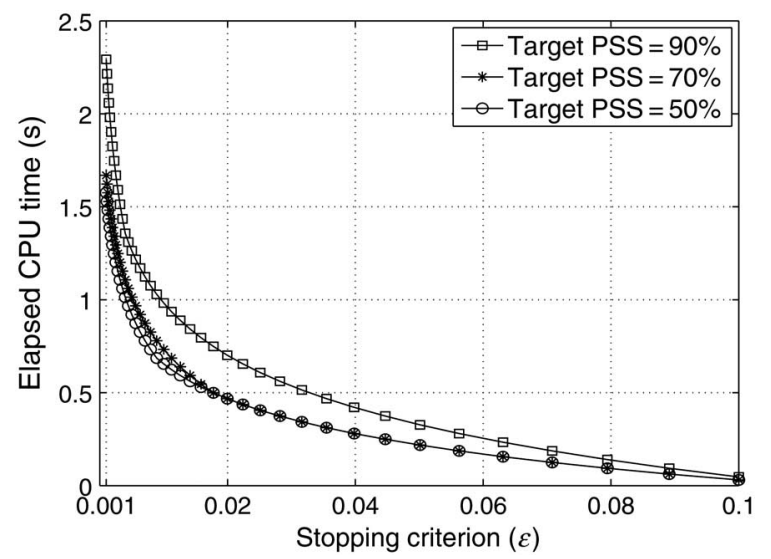

Fig. 3. Elapsed CPU time of the proposed algorithm under different stopping criteria. The computer used was an ThinkPad Laptop with a i7 M560 duo-core processor at $2.67 \mathrm{GHz}$.

order of $\mathbf{O}\left(\beta^{N} N \log (1 / \epsilon)\right)$, with $1<\beta<2$ [34], and a simple GA involves a complexity of $\mathbf{O}$ (gens $\cdot \operatorname{pop} \cdot 2 N)$, where gens is the number of generations and pop the population size [35]. Therefore, the proposed method achieves accurate results with lower computational complexity compared to BB and GA as shown in Fig. 2. The corresponding CPU time to run the proposed algorithm has been plotted in Fig. 3. It is observed that the algorithm running time decreases as the stopping criterion drops from within $0.1 \%$ of the value in the previous iteration to $10 \%$.

The optimal amount of dispatch of each DG for given demand and wind power forecast profiles is shown in Fig. 4 under different target PSS values. Take Fig. 4(b) as an example. Observe the forecast wind power decreases from 1:00 A.M. to 5:00 A.M. in the early morning. In order to fulfill the demand, the DGs are dispatched economically according to the optimal UC solution. The dispatched power from the DGs continues to be in a higher level until 13:00 P.M., when the forecast wind power comes into play again. At midnight, the power demand reaches a minimum and the microgrid is entirely powered by the wind power. During the whole process, the DGs serve as backup 


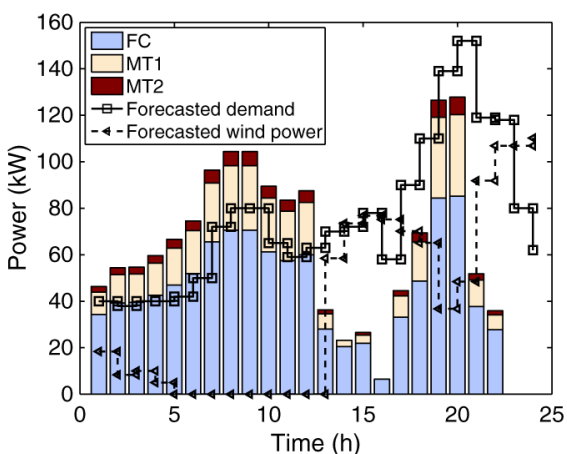

(a)

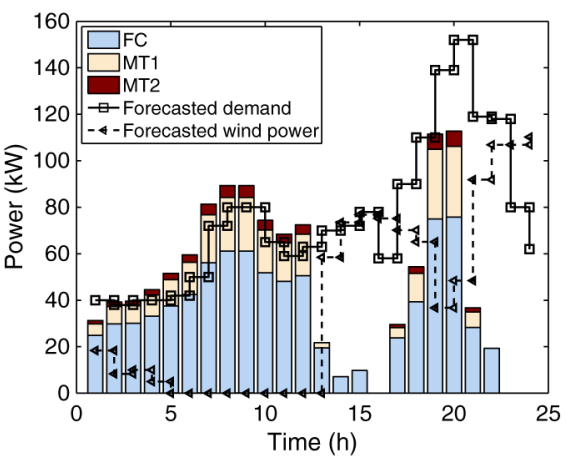

(b)

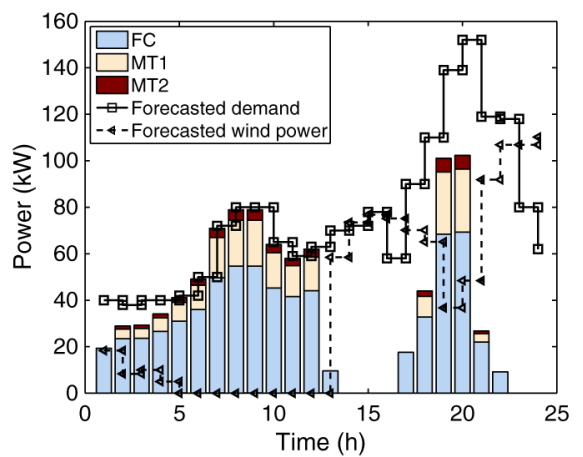

(c)

Fig. 4. Forecasted demand and wind power as well as optimal dispatch of the DGs under different PSS targets: (a) target PSS $=90 \%$, (b) target PSS $=70 \%$, and target PSS $=50 \%$.

TABLE II

Unit Commitment of the Distributed Generators with Target PSS $=70 \%$

\begin{tabular}{|c|c|}
\hline Unit & Hours (1-24) \\
\hline MT1 & 1111111111111000011111 \\
\hline MT2 & 111111111111000011111000 \\
\hline & 11111111111111111110111111100 \\
\hline
\end{tabular}

sources that complement the renewable source in meeting electricity demand.

Table II further depicts the UC status of the DGs for the case of PSS $=70 \%$ over a period of $24 \mathrm{~h}$. Cross-referencing Fig. 4(b), we can observe that an $\mathrm{FC}$ is the most preferred power source among the DGs and contributes significantly during the entire scheduling period. In contrast, MT2 contributes the least and is always the last one to be turned on. The reason is as follows. Recall from (18) in Section III-B that the commitment status of the DG is solely determined by the sign of the UC indication function $\varphi_{i, t}($.$) at the optimal dispatched point p_{i, t}^{*}$. An interesting phenomenon observed from numerical results is that $\varphi_{3, t}\left(p_{3, t}^{*}\right)<\varphi_{1, t}\left(p_{1, t}^{*}\right)<\varphi_{2, t}\left(p_{2, t}^{*}\right)$ for all $t=1, \ldots, T$. In other words, there exists a hidden priority listing order that arranges the DGs based on lowest operational cost characteristics as well as emissions.

\section{B. Operating Cost Versus Different Forecasting Time Horizons}

Clearly, the shorter the forecasting interval is, the more accurate the forecast data and the smaller the variance of the forecast error. According to [29] and [36], the typical standard deviation of the wind power forecast error for a specific wind farm can be expressed as a function of the forecast horizon, which can be approximated accurately by a linear function when the forecast horizon is less than $6 \mathrm{~h}$. Fig. 5 examines the impact of varied forecasting time horizons on the total operating cost, based on the aforementioned linear model. It is observed that for the case of PSS $=90 \%$, the operating cost grows almost linearly as the forecasting interval increases from $10 \mathrm{~min}$ to $1 \mathrm{~h}$. The same trend is observed for the case of PSS $=70 \%$. Interestingly, the cost holds constant for the case of PSS $=50 \%$, due to the fact that the microgrid is indifferent to either being connected to, or autonomous of the macrogrid.

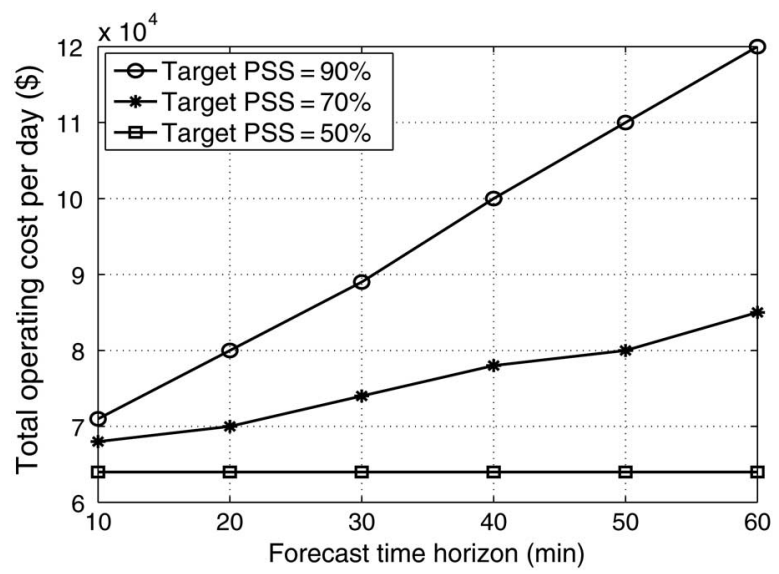

Fig. 5. Total operating cost per day under different time horizons.

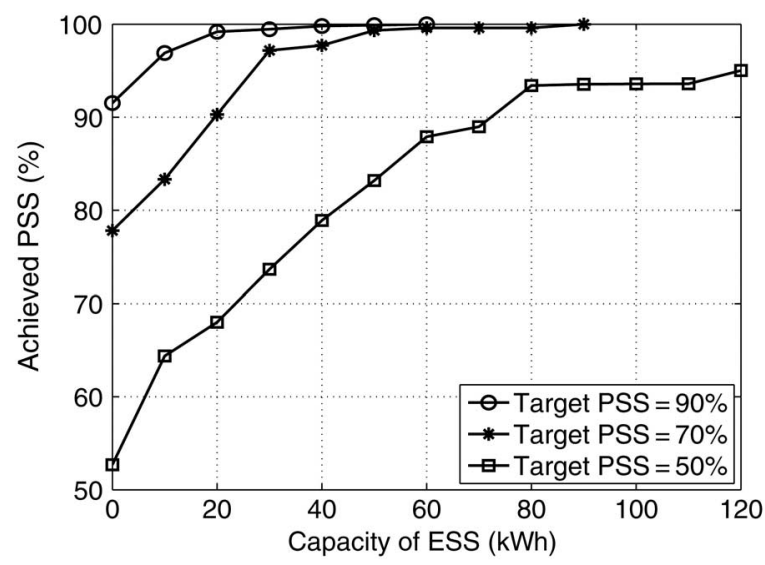

Fig. 6. Achieved PSS versus the capacity of the ESS based on wind power statistics collected in 1 month.

\section{The Impact of ESS on Microgrid's Autonomy}

In the last numerical example, we incorporate the ESS into the microgrid setting and study the impact of ESS on the achieved level of autonomy of the microgrid. To verify the effectiveness of the ESS on the achieved PSS, data samples collected by the wind test center [33] in 1 month are employed to calculate the achieved PSS of the microgrid in practice. We observe from Fig. 6 that for 


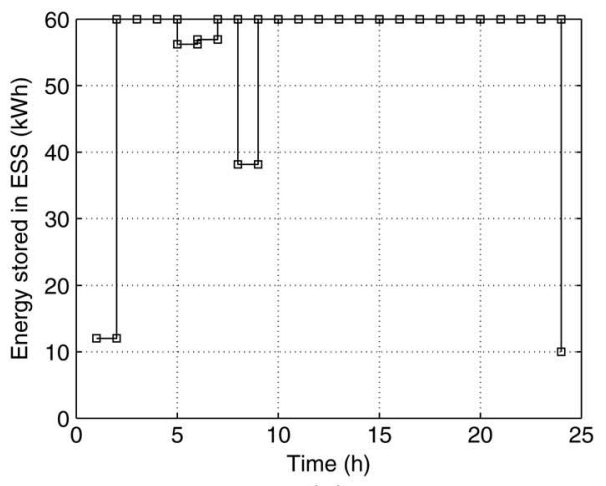

(a)

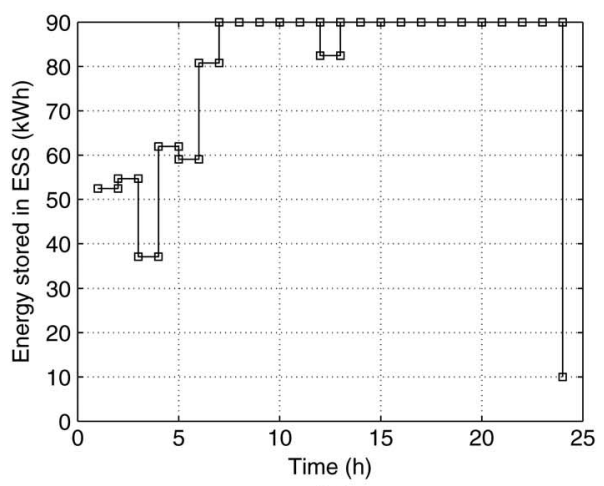

(b)

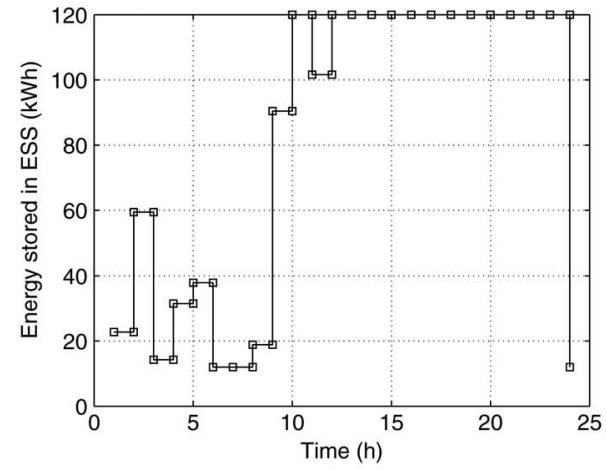

(c)

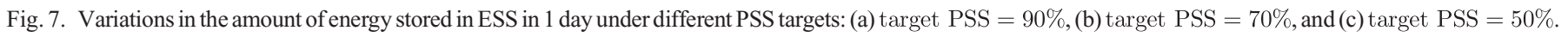

all the PSS targets, 1) the proposed approach successfully meets the design targets in the absence of ESS. For example, the microgrid achieves a practical PSS of $52 \%, 78 \%$, and $92 \%$ when the PSS targets are set as $50 \%, 70 \%$, and $90 \%$, respectively; 2 ) the microgrid is more capable of functioning self-sufficiently as the capacity of ESS increases [37]; and 3) the achieved PSS hits an upper limit when the capacity of ESS is larger than a threshold. From our simulations, this ESS threshold grows from $40 \mathrm{kWh}$, to $90 \mathrm{kWh}$, and to $120 \mathrm{kWh}$ as the PSS targets decreases from $90 \%$ to $70 \%$, and to $50 \%$, respectively. This provides a guideline on determining the ESS size to achieve a desired PSS level.

To further analyze the variations of the energy stored in the ESS in 1 day, we plot in Fig. 7 the evolution of the energy stored in ESS under different target PSSs. The capacity of ESS is set as 60, 90, and $120 \mathrm{kWh}$ as in Fig. 7(a), (b), and (c) when the PSS targets are equal to $90 \%, 70 \%$, and $50 \%$, respectively. Due to practical concerns, the starting $(t=0)$ and ending limits $(t=24)$ of ESS in this work are set as around $10 \%$ of the capacity of ESS, which are $10 \mathrm{kWh}$ for all the cases. We then observe that the events of charging and discharging occur frequently during the time period between 0:00 A.M. and 10:00 A.M., when the forecasted wind power falls short of the forecasted demand. Also note that the stored energy in the ESS varies less remarkably as the microgrid tends to operate more independently.

\section{CONCLUSION}

The unique characteristics of renewable-powered microgrid have brought new challenges to the classic UC optimization task of UC. We have shown that the traditional problem formulation can be modified to incorporate the intermittency of the RESs, emission limits on the carbon footprint, as well as the forecast error that exists in demand and renewable power forecasts. Using a duality-based approach, it has been demonstrated that an analytical characterization of the optimal commitment and dispatch solutions for the DGs is available, which can be computed very efficiently using a subgradient-based algorithm. The approach can be easily modified to incorporate other types of DGs or RESs. Our study shows that the features of DGs can have a great impact on the operation of UC strategy in microgrids, which will be investigated in our future work.

\section{REFERENCES}

[1] V. C. Güngör, D. Sahin, T. Kocak, S. Ergüt, C. Buccella, C. Cecati, and G. P. Hancke, "Smart grid technologies: Communications technologies and standards," IEEE Trans. Ind. Inform., vol. 7, no. 4, pp. 529-539, Nov. 2011.

[2] A. K. Basu, S. Chowdhury, and S. Paul, "Microgrids: Energy management by strategic deployment of DERs-A comprehensive survey," Renew. Sustain. Energy Rev., vol. 15, no. 9, pp. 4348-4356, Dec. 2011.

[3] A. Cohen and V. Sherkat, "Optimization-based methods for operations scheduling," Proc. IEEE, vol. 75, no. 12, pp. 1574-1591, Dec. 1987.

[4] N. Padhy, "Unit commitment-A bibliographical survey," IEEE Trans. Power Syst., vol. 19, no. 2, pp. 1196-1205, May 2004.

[5] S. A. Pourmousavi, M. H. Nehrir, C. M. Colson, and C. Wang, "Real-time energy management of a stand-alone hybrid wind-microturbine energy system using particle swarm optimization," IEEE Trans. Sustain. Energy, vol. 1, no. 3, pp. 193-201, Oct. 2011.

[6] R. Yinger, "Behavior of Capstone and Honeywell microturbine generators during load changes," California Energy Commission, Tech. Rep. P50004-021, 2001.

[7] C. Hernandez-Aramburo, T. Green, and N. Mugniot, "Fuel consumption minimization of a microgrid," IEEE Trans. Ind. Appl., vol. 41, no. 3, pp. 673-681, May 2005.

[8] C. Chen, S. Duan, T. Cai, B. Liu, and G. Hu, "Smart energy management system for optimal microgrid economic operation," IET Renew. Power Gen., vol. 5, no. 3, pp. 258-267, May 2011.

[9] T. Logenthiran and D. Srinivasan, "Short term generation scheduling of a microgrid," in Proc. IEEE TENCON, Singapore, Nov. 2009, pp. 1-6.

[10] M. R. Alrashidi and M. E. El-Hawary, "Impact of loading conditions on the emission-economic dispatch," World Academy of Science, Eng. and Technol., vol. 15, no. 29, pp. 148-151, Mar. 2008.

[11] R. Brown, S. Gupta, R. Christie, S. Venkata, and R. Fletcher, "Automated primary distribution system design: Reliability and cost optimization," in Proc. IEEE Transmission and Distribution Conf., Los Angeles, CA, USA, Nov. 1996.

[12] S. Jonnavithula and R. Billinton, "Minimum cost analysis of feeder routing in distribution system planning," IEEE Trans. Power Del., vol. 11, no. 4, pp. 1935-1940, Oct. 1996.

[13] Y. Tang, "Power distribution system planning with reliability modeling and optimization," IEEE Trans. Power Syst., vol. 11, no. 1, pp. 181-189, Feb. 1996.

[14] S. Chen, H. Gooi, and M. Wang, "Sizing of energy storage for microgrids," IEEE Trans. Smart Grid, vol. 3, no. 1, pp. 142-151, Mar. 2012.

[15] A. Abdollahi, M. P. Moghaddam, M. Rashidinejad, and M. K. Sheikh-ElEslami, "Investigation of economic and environmental-driven demand response measures incorporating UC," IEEE Trans. Smart Grid, vol. 3, no. 1 , pp. 12-25, Mar. 2012.

[16] A. Hawkes and M. Leach, "Modelling high level system design and unit commitment for a microgrid," Appl. Energy, vol. 86, no. 7-8, pp. 1253-1265, Jul. 2009.

[17] I. Gradshteyn and I. Ryzhik, in Table of Integrals, Series, and Products, A. Jeffrey and D. Zwillinger, Eds., 7th ed., New York, NY, USA: Elsevier Academic Press, 2007.

[18] H. Feroze, "Multi-agent systems in microgrids: Design and implementation," Master Thesis, Virginia Tech, 2009.

[19] L. Tao and C. Schwaegerl, "Advanced architectures and control concepts for more microgrids,” EC Project, Tech. Rep. SES6-019864, 2009. 
[20] M. Pipattanasomporn, H. Feroze, and S. Rahman, "Securing critical loads in a PV-based microgrid with a multi-agent system," Renew. Energy, vol. 39, no. 1, pp. 166-174, Mar. 2012.

[21] J. Carta, P. Ramrez, and S. Velázquez, "A review of wind speed probability distributions used in wind energy analysis," Renew. Sustain. Energy Rev., vol. 13, no. 5, pp. 933-955, Jun. 2009.

[22] S. Roy, "Market constrained optimal planning for wind energy conversion systems over multiple installation sites," IEEE Trans. Energy Convers., vol. 17 , no. 1, pp. 124-129, Mar. 2002.

[23] F. Barbir and T. Gómez, "Efficiency and economics of proton exchange membrane fuel cells," Int. J. Hydrogen Energy, vol. 13, no. 8, pp. 1027-1037, 1997.

[24] F. Mohamed, "Microgrid modelling and online management," Ph.D. dissertation, Helsinki University of Technology, Helsinki, Finland, 2008.

[25] A. Azmy and I. Erlich, "Online optimal management of PEM fuel cells using neural networks," IEEE Trans. Power Del., vol. 20, no. 2, pp. 1051-1058, Apr. 2005

[26] J. L. Bernal-Agustn and R. Dufo-López, "Economical and environmental analysis of grid connected photovoltaic systems in Spain," Renew. Energy, vol. 31 , no. 8, pp. 1107-1128, Jul. 2006.

[27] F. Mohamed and H. Koivo, "Multiobjective optimization using modified game theory for online management of microgrid," Eur. Trans. Electr. Power, vol. 21, no. 1, pp. 839-854, 2011.

[28] A. Saber and G. Venayagamoorthy, "Plug-in vehicles and renewable energy sources for cost and emission reductions," IEEE Trans. Ind. Electron., vol. 58, no. 4, pp. 1229-1238, Apr. 2011.

[29] R. Doherty and M. O'Malley, "A new approach to quantify reserve demand in systems with significant installed wind capacity," IEEE Trans. Power Syst., vol. 22, no. 2, pp. 587-595, May 2005.

[30] S. Tewari, C. J. Geyer, and N. Mohan, "A statistical model for wind power forecast error and its application to the estimation of penalties in liberalized markets," IEEE Trans. Power Syst., vol. 26, no. 4, pp. 2031-2039, Nov. 2011.

[31] S. Boyd and L. Vandenberghe, Convex Optimization, Cambridge, U.K.: Cambridge Univ. Press, 2004

[32] D. P. Bertsekas, Nonlinear Programming, Belmont, MA, USA: Athena Scientific, 1995

[33] "Wind energy database of white deer" [Online]. Available: http://www. windenergy.org/datasites/14-whitedeer/.

[34] W. Zhang, "Branch-and-Bound search algorithms and their computational complexity," University of Southern California Marina Delrey Information Sciences Inst, Tech. Rep. ADA314598, May 1996.

[35] "What is time complexity of genetic algorithm" [Online]. Available: http:// wiki.answers.com/Q/What-is-time-complexity-of-genetic-algorithm.

[36] D. Lew and M. Milligan, "The value of wind power forecasting," in Proc. 91st American Meteorological Society Annual Meeting, Seattle, WA, USA, Jan. 2011.

[37] S. Arefifar, Y. Mohamed, and T. El-Fouly, "Supply-adequacy-based optimal construction of microgrids in smart distribution systems," IEEE Trans. Smart Grid, vol. 3, no. 3, pp. 1491-1502, Sep. 2012.

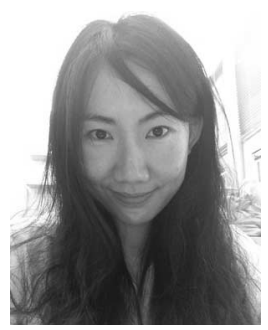

Binyan Zhao (S'13) received the B.Sc. and M.Sc. degrees in telecommunication from Beijing University of Posts and Telecommunications, Beijing, China, in 2008 and 2011, respectively. She is currently working toward the Ph.D. degree at the Department of Electrical and Computer Engineering, University of Victoria, Victoria, BC, Canada

Her research interests include scheduling problems in distribution power network and service restoration issues in microgrid.

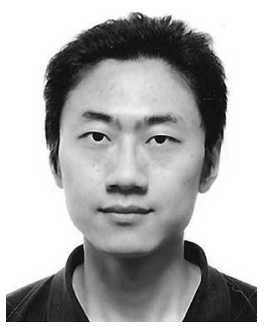

Yi Shi (S'06-M'10) received the B.E. degree in telecommunications from Beijing University of Posts and Telecommunications, Beijing, China, in 2006. He received the $\mathrm{Ph} . \mathrm{D}$. degree in electronic and computer engineering from The Hong Kong University of Science and Technology, Hong Kong, in August 2010.

From 2010 to 2012, he worked as a post-doctoral fellow in the Department of Electrical and Computer Engineering, University of Victoria. He is currently an Engineer with Huawei Technologies, Beijing, China. His work involves the $3 \mathrm{GPP}$ standarization of the next-generation wireless mobile communication systems. His research interests include cooperative communications, resource allocation and scheduling using optimization and game theory, etc.

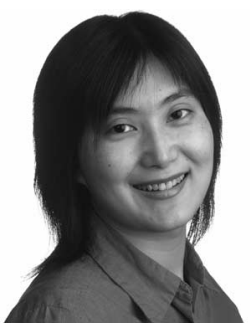

Xiaodai Dong (S'97-M'00-SM'09) received the B.Sc. degree in information and control engineering from Xi'an Jiaotong University, Xi'an, China, the M.Sc. degree in electrical engineering from National University of Singapore, Singapore, and the Ph.D. degree in electrical and computer engineering from Queen's University, Kingston, ON, Canada, in 1992, 1995, and 2000, respectively.

She is currently a Professor and Canada Research Chair (Tier II) with the Department of Electrical and Computer Engineering, University of Victoria, Victoria, BC, Canada. Between 2002 and 2004, she was an Assistant Professor with the Department of Electrical and Computer Engineering, University of Alberta, Edmonton, AB, Canada. From 1999 to 2002, she was with Nortel Networks, Ottawa, ON, where she worked on the base transceiver design of third-generation mobile communication systems. Her research interests include communication theory, radio propagation, cooperative communications, ultrawideband radio, and signal processing for communication applications.

Dr. Dong is an Editor for the IEEE Transactions on Wireless Communications, the IEEE Transactions on Vehicular Technology, and the Journal of Communications and Networks.

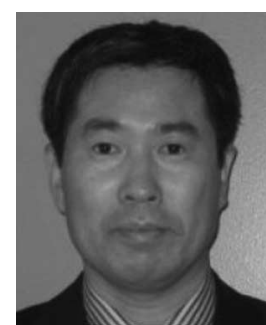

Wenpeng Luan (SM'05) received the B.Sc. degree from Tsinghua University, Beijing, China, in 1986, the M.Sc. degree from Tianjin University, Tianjin, China, in 1989, and the Ph.D. degree from Strathclyde University, Glasgow, U.K., in 1999, all in electrical engineering.

He was an Engineering Team Leader in the Smart Metering and Infrastructure Program at BC Hydro, Burnaby, BC, Canada. He is currently working at the State Grid Smart Grid Research Institute, Beijing, China. His special fields of interest include smart metering, smart grid solutions, system planning, asset management, control system cyber security, and utility advanced applications.

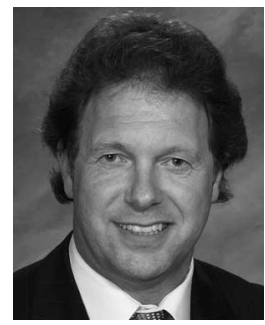

Jens Bornemann (M'87-SM'90-F'02) received the Dipl.-Ing. and the Dr.-Ing. degrees, both in electrical engineering, from the University of Bremen, Germany, in 1980 and 1984, respectively.

From 1984 to 1985 , he was a Consulting Engineer. In 1985, he joined the University of Bremen, Germany, as an Assistant Professor. Since April 1988, he has been with the Department of Electrical and Computer Engineering, University of Victoria, Victoria, BC, Canada, where he became a Professor in 1992. From 1992 to 1995 , he was a Fellow of the British Columbia Advanced Systems Institute. In 1996, he was a Visiting Scientist at Spar Aerospace Limited (now MDA Space), Ste-Anne-de-Bellevue, Qubec, Canada, and a Visiting Professor at the Microwave Department, University of Ulm, Germany. In 2003, he was a Visiting Professor at the Laboratory for Electromagnetic Fields and Microwave Electronics, ETH Zurich, Switzerland. From 1997 to 2002, he was a codirector of the Center for Advanced Materials and Related Technology (CAMTEC), University of Victoria. From 1999 to 2002, he served as an Associate Editor of the IEEE Transactions on Microwave Theory AND TECHNIQUes in the area of microwave modeling and CAD. From 2006 to 2008, he was an Associate Editor of the International Journal of Electronics and Communications. From 1999 to 2009, he served on the Technical Program Committee of the IEEE MTT-S International Microwave Symposium. He has coauthored Waveguide Components for Antenna Feed Systems-Theory and Design (Artech House, 1993) and has authored/coauthored more than 250 technical papers. His research activities include RF/wireless/microwave/millimeterwave components and systems design, and field-theory-based modeling of integrated circuits, feed networks, ultrawideband technology, and antennas.

Dr. Bornemann is a Registered Professional Engineer in the Province of British Columbia, Canada. He is a Fellow of the Canadian Academy of Engineering (CAE) and serves on the editorial advisory board of the International Journal of Numerical Modeling. 